\title{
TRIM24 promotes the aggression of gastric cancer via the Wnt/ $\beta$-catenin signaling pathway
}

\author{
ZILING FANG* ${ }^{*}$ JUN DENG ${ }^{*}$, LING ZHANG, XIAOJUN XIANG, \\ FENG YU, JUN CHEN, MIAO FENG and JIANPING XIONG
}

\begin{abstract}
Department of Oncology, The First Affiliated Hospital of Nanchang University, Nanchang, Jiangxi 330006, P.R. China
\end{abstract}
Received July 7, 2015; Accepted November 3, 2016

DOI: $10.3892 / \mathrm{ol} .2017 .5604$

\begin{abstract}
Tripartite motif-containing 24 (TRIM24) is important in tumor development and progression. However, the role of TRIM24 in gastric cancer (GC) and the mechanisms underlying the dysregulated expression of TRIM24 remain to be fully elucidated. In the present study, it was found that TRIM24 was frequently overexpressed in GC cell lines and tissues compared with normal controls, as determined by western blotting and immunohistochemical staining. The high nuclear expression of TRIM24 was correlated with the depth of invasion $(\mathrm{P}=0.007)$, tumor-node-metastasis stage $(\mathrm{P}=0.005)$, and lymph node metastasis $(\mathrm{P}=0.027)$, and shorter overall survival rates $(\mathrm{P}=0.010)$ in patients with $\mathrm{GC}$. Small interfering RNA-mediated knockdown of TRIM24 inhibited cell proliferation, colony formation, migration, invasion and the nuclear accumulation of $\beta$-catenin, and it delayed cell cycle progression and induced apoptosis. In addition, the expression of TRIM24 was positively correlated with that of $\beta$-catenin in GC tissues. TRIM24 knockdown decreased the expression of $\mathrm{Wnt} / \beta$-catenin target genes, whereas the activation of Wnt/ $\beta$-catenin signaling by lithium chloride reversed the effects of TRIM24 knockdown. Taken together, these data suggested that TRIM24 was a prognostic or potential therapeutic target for patients with GC and was important in the activation of the $\mathrm{Wnt} / \beta$-catenin pathway during the progression of GC.
\end{abstract}

\section{Introduction}

Gastric cancer (GC) is the second leading cause of cancer-associated mortality worldwide (1). Despite substantial advances

Correspondence to: Professor Jianping Xiong, Department of Oncology, The First Affiliated Hospital of Nanchang University, 17 Yongwaizheng Road, Nanchang, Jiangxi 330006, P.R. China E-mail: jpxiong@medmail.com.cn

${ }^{*}$ Contributed equally

Key words: tripartite motif-containing 24, gastric cancer, aggressiveness, Wnt/ $\beta$-catenin in surgery, chemo- and radiotherapy, and targeted molecular therapy, the 5-year survival rate of patients with advanced GC remains low. Therefore, identifying novel diagnostic and prognostic markers, and elucidating the mechanisms underlying disease progression are essential for preventing and treating gastric tumorigenesis.

The Wnt/ $\beta$-catenin signaling pathway is implicated in oncogenesis, and contributes to the initiation and progression of GC (2). In the absence of Wnt ligands, cytoplasmic $\beta$-catenin is phosphorylated by a multi-protein complex consisting of adenomatous polyposis coli (APC), axin, casein kinase 1 and glycogen synthase kinase (GSK)-3 $\beta$, leading to its ubiquitination and degradation $(3,4)$. This is inhibited upon activation of Wnt signaling; $\beta$-catenin accumulates in the cytoplasm and translocates to the nucleus, where it initiates the transcription of a variety of target genes. The accumulation of nuclear $\beta$-catenin is a hallmark of Wnt signaling activation (5). Somatic mutations in APC, $\beta$-catenin and axin, among other factors, are responsible for activating the Wnt/ $\beta$-catenin pathway (6-8). However, the mechanisms by which the $\mathrm{Wnt} / \beta$-catenin pathway is activated in $\mathrm{GC}$ remain to be fully elucidated.

The tripartite motif (TRIM) family, identified as a subfamily of the RING-type E3 ubiquitin ligase family, is involved in a broad range of biological processes, including cell growth and apoptosis, development and tumorigenesis (9). TRIM24, formerly known as transcriptional intermediary factor $1 \alpha$, is a member of the TRIM family, which is characterized by a RING domain, two B-box zinc fingers and a coiled-coin region (10). The aberrant overexpression of TRIM24 is a prognostic factor in several types of cancer, and promotes tumor development and progression through various mechanisms. TRIM24 ubiquitinates and induces the proteasome-mediated degradation of p53 $(11,12)$, and can also bind to chromatin and the estrogen receptor to activate target genes associated with cell proliferation and tumor development in breast cancer (13). TRIM24 also promotes tumorigenesis by activating aerobic glycolysis (14) and serves as a target of chromosomal translocations leading to the formation of oncogenic fusion proteins (15-17). However, the role of TRIM24 in the development of GC and the underlying molecular mechanisms remain to be fully elucidated. Previous studies have indicated that TRIM proteins regulate $\mathrm{Wnt} / \beta$-catenin signaling. TRIM29 promotes proliferation and metastasis via Wnt/ $\beta$-catenin 
pathway activation in pancreatic cancer (18), whereas TRIM24 knockdown in human HepG2 liver cancer cells downregulates $\beta$-catenin and cyclinD1, two major downstream genes of the Wnt pathway (19). Therefore, the present study hypothesized that TRIM24 promotes the aggressiveness of GC by activating Wnt/ $\beta$-catenin signaling.

To confirm this hypothesis, the present study examined the expression and functions of TRIM24 in GC cell lines and tissue samples. It was found that TRIM24 was upregulated in GC, which was positively correlated with the expression of $\beta$-catenin. In addition, TRIM24 knockdown suppressed cell proliferation, arrested cells at the G0/G1 phase, inhibited migration, invasion and the nuclear translocation of $\beta$-catenin, and induced apoptosis. It was confirmed that TRIM24 exerted its oncogenic functions through activation of the Wnt/ $\beta$-catenin pathway. Therefore, the findings of the present study indicated that TRIM24 may serve as a potential therapeutic target for $\mathrm{GC}$ and is important in activation of the $\mathrm{Wnt} / \beta$-catenin pathway during the progression of GC.

\section{Materials and methods}

Ethics statement. The present study was approved by the Ethics Committee of the First Affiliated Hospital of Nanchang University (Nanchang, China). Informed consent was provided by patients from whom tissue samples were obtained for investigation.

Cell lines and culture. The human MGC803 and SGC7901 GC cell lines were purchased from the Type Culture Collection of the Chinese Academy of Science (Shanghai, China). GES-1, a normal human gastric mucosa cell line, and the AGS, BGC823 and HGC-27 GC cell lines were obtained from the Sun Yat-Sen University Cancer Center (Guangzhou, China). All cells were cultured in Roswell Park Memorial Institute (RPMI) 1640 medium (HyClone; GE Healthcare Life Sciences, Logan, UT, USA) supplemented with $10 \%$ fetal bovine serum (FBS; HyClone; GE Healthcare Life Sciences) for $24 \mathrm{~h}$ in a humidified chamber at $37^{\circ} \mathrm{C}$ and $5 \% \mathrm{CO}_{2}$.

Patients and tissue samples. A total of 4 fresh GC tissues and paired noncancerous gastric mucosal tissues were collected by gastrectomy from the Department of General Surgery of the First Affiliated Hospital of Nanchang University. All the fresh samples were immediately snap-frozen in liquid and stored at $-80^{\circ}$. Paraffin-embedded GC tissues $(n=90)$ and gastric normal tissues $(n=60)$ were collected from the Department of Pathology of the First Affiliated Hospital of Nanchang University between 2007 and 2009. None of these 90 patients with GC had received neoadjuvant chemotherapy or radiation therapy prior to surgery. The overall survival (OS) of the patients with GC was defined as the period between the date of surgery to the time at which the patient succumbed to mortality from any cause, or the date of the last follow-up if no event was documented. Tumor stage was determined according to the 2010 American Joint Committee on Cancer criteria (20). Histological differentiation was based on World Health Organization criteria (21). The detailed clinical information of the patients is summarized in Table I.
Immunohistochemistry. The tissue samples were formalin-fixed overnight and then paraffin-embedded at room temperature, prior to being cut into $4-\mu \mathrm{m}$ serial sections. The sections were deparaffinized and rehydrated by incubation in 3\% hydrogen peroxide for $15 \mathrm{~min}$ at room temperature. Antigen retrieval was performed by heating the sections in citrate buffer at $95^{\circ} \mathrm{C}$ for $1 \mathrm{~h}$. The sections were then incubated with rabbit anti-human polyclonal TRIM24 antibody (1:200 dilution; catalog no. , ab70560; Abcam, Cambridge, UK) or rabbit antihuman monoclonal $\beta$-catenin antibody (1:100 dilution; catalog no. 8480; Cell Signaling Technology, Inc., Danvers, MA, USA) in a humidified chamber overnight at $4^{\circ} \mathrm{C}$. Following washing three times with phosphate-buffered saline (PBS), the sections were incubated at room temperature with a horseradish peroxidase system and DAB substrate (Dako, Carpinteria, CA, USA), followed by incubation with PBS containing diaminobenzidine for $10 \mathrm{~min}$ at room temperature.

The tissue samples were examined by two investigators who were blinded to the pathological information, and immunoreactivity was scored using the German Semi-Quantitative method (22). For TRIM24 staining, each specimen was scored according to its staining intensity $(0$, none; 1 , weak; 2 , moderate; 3 , strong) and the percentage of stained cells $(0,0 \% ; 1,1-24 \%$; $2,25-49 \% ; 3,50-74 \% ; 4,75-100 \%)$. A final staining index (SI) was calculated as the product of the intensity and percentage scores, yielding a value between 0 and 12, which was classified as negative $(-, 0-1)$, weak $(+; 2-4)$, moderate $(++; 6-8)$ or strong (+++; 9-12). An SI $\geq 6$ was considered a high expression of TRIM2 4 and an SI<6 was considered a low expression of TRIM24. For $\beta$-catenin, membrane or cytoplasmic staining was considered negative, whereas nuclear expression was considered positive. The scoring of TRIM 24 and $\beta$-catenin were evaluated independently by two board-certified clinical pathologists blinded to the clinical parameters. Any discrepancy between the scores assigned by the two investigators was resolved by re-evaluation and careful discussion until a consensus was reached.

Reverse transcription-quantitative polymerase chain reaction (RT-qPCR) analysis. Total RNA was extracted with TRIzol reagent (Invitrogen; Thermo Fisher Scientific, Inc., Waltham, MA, USA) and RNA concentration was measured using spectrophotometry. cDNA was obtained using an EasyScript First-Strand cDNA Synthesis Supermix kit (TransGen Biotech, Inc., Beijing, China) and RT-qPCR analysis was performed on an ABI Prism 7500 Sequence Detection system (Applied Biosystems; Thermo Fisher Scientific, Inc.) using SYBR Green qPCR Supermix-UDG with ROX (Invitrogen; Thermo Fisher Scientific, Inc.) according to the manufacturer's protocol. The primers used for RT-qPCR analysis are listed in Table II. The target gene expression levels were was normalized against that of the housekeeping gene, glyceraldehyde-3-phosphate dehydrogenase, and calculated as $2^{-[(\mathrm{Cq} \text { target gene) -(Cq GAPDH)] }}$, where $\mathrm{Cq}$ is the quantification cycle for each transcript (23).

Western blot analysis. The cells and tissues samples were lysed in radioimmunoprecipitaion assay buffer (Sigma-Aldrich; Merck Millipore, Darmstadt, Germany) supplemented with protease in inhibitor cocktail (Roche Diagnostics $\mathrm{GmbH}$, Mannheim, Germany). The protein concentration was 
Table I. Association between the expression of TRIM24 and clinicopathological parameters of patients with gastric cancer.

\begin{tabular}{|c|c|c|c|c|}
\hline \multirow[b]{2}{*}{ Variable } & \multirow[b]{2}{*}{$\mathrm{n}$} & \multicolumn{2}{|c|}{ Expression of TRIM24 } & \multirow[b]{2}{*}{ P-value } \\
\hline & & Low & High & \\
\hline Age (years) & & & & 0.848 \\
\hline$<60$ & 50 & 21 & 29 & \\
\hline$\geq 60$ & 40 & 16 & 24 & \\
\hline Gender & & & & 0.294 \\
\hline Male & 55 & 25 & 30 & \\
\hline Female & 35 & 12 & 23 & \\
\hline Tumor size $(\mathrm{cm})$ & & & & 0.571 \\
\hline$<5$ & 47 & 18 & 29 & \\
\hline$\geq 5$ & 43 & 19 & 24 & \\
\hline Tumor location & & & & 0.605 \\
\hline Proximal & 32 & 12 & 20 & \\
\hline Distal & 58 & 25 & 33 & \\
\hline Depth of invasion ${ }^{a}$ & & & & $0.007^{\mathrm{c}}$ \\
\hline $\mathrm{T}_{1}-\mathrm{T}_{2}$ & 42 & 23 & 19 & \\
\hline $\mathrm{T}_{3}-\mathrm{T}_{4}$ & 48 & 13 & 35 & \\
\hline Differentiation & & & & 0.176 \\
\hline Well or moderately & 41 & 20 & 21 & \\
\hline Poor & 49 & 17 & 32 & \\
\hline Lauren classification & & & & 0.551 \\
\hline Intestinal type & 34 & 13 & 21 & \\
\hline Diffuse type & 56 & 22 & 34 & \\
\hline TNM stage $^{a}$ & & & & $0.005^{\mathrm{c}}$ \\
\hline I-II & 43 & 27 & 16 & \\
\hline III-IV & 47 & 10 & 37 & \\
\hline Lymph node metastasis & & & & $0.027^{\mathrm{b}}$ \\
\hline $\mathrm{N}_{0}$ & 39 & 21 & 18 & \\
\hline $\mathrm{N}_{\mathrm{X}}$ & 51 & 16 & 35 & \\
\hline
\end{tabular}

${ }^{a}$ Depth of tumor invasion and TNM stage according to the 2010 TNM Classification of Malignant Tumors by the International Union Against Cancer; ${ }^{\mathrm{b}} \mathrm{P}<0.05$; ${ }^{\mathrm{c}} \mathrm{P}<0.01$. $\mathrm{P}$-values determined using $\chi^{2}$ test. TRIM24, tripartite motif-containing 24; TNM, tumor node metastasis.

determined by Bradford method with bovine serum albumin (BSA) as the standard. Equal quantities of protein $(50 \mu \mathrm{g})$ from each group were separated by $8 \%$ sodium dodecyl sulfate-polyacrylamide gel electrophoresis and transferred onto a polyvinylidene difluoride membrane. Following incubation in blocking buffer for $1 \mathrm{~h}$ at room temperature, the membrane was incubated overnight at $4^{\circ} \mathrm{C}$ with the following primary antibodies: Rabbit anti-human polyclonal TRIM24 (1:1,000 dilution; catalog no. ab70560; Abcam), rabbit antihuman monoclonal $\beta$-catenin (1:1,000 dilution; catalog no. 8480; Cell Signaling Technology, Inc.), rabbit anti-human monoclonal c-Myc (1:1,000 dilution; catalog no. 5605; Cell Signaling Technology, Inc.), rabbit anti-human monoclonal cyclinD1 (1:1,000 dilution; catalog no. 2978; Cell Signaling Technology, Inc.) and mouse anti-human monoclonal $\beta$-actin (1:2,000; catalog no. 8H10D10; Cell Signaling Technology, Inc.). Following three washes in Tris-buffered saline with $0.1 \%$ Tween 20 , the membrane was incubated with horseradish peroxidase-labeled anti-rabbit or anti-mouse secondary antibody (1:3,000 dilution; Santa Cruz Biotechnology, Inc., Dallas, TX, USA) for $1 \mathrm{~h}$ at room temperature and developed using a chemiluminescence detection system (Thermo Fisher Scientific, Inc.).

Cell transfection. Small interfering RNAs (siRNAs) targeting TRIM24 and negative control (NC) siRNA were purchased from Shanghai GenePharma Co., Ltd. (Shanghai, China). The TRIM24 siRNA sequence was 5'-GCUGGA CUCUCUAAACAAUTT-3'. In vitro transient transfection was performed using Lipofectamine 2000 (Invitrogen; Thermo Fisher Scientific, Inc.) according to the manufacturer's protocol. The cells were divided into three groups: Blank, transfected with NC siRNA or transfected with TRIM24 siRNA, and grown to $70-80 \%$ confluency prior to transfection. TRIM24-knockdown was confirmed using RT-PCR and western blot analyses. To examine the effects 
Table II. Sequences of primers for reverse transcription-quantitative polymerase chain reaction analysis.

Gene Primer

TRIM24 Forward: 5'-CATATGCAGCAACAGCAACCG-3' Reverse: 5'- GAAAGCCATCTGTAGGGGGT-3'

$\beta$-catenin Forward: 5'-GGATCAAACCTGACAGCCA-3' Reverse: 5'-GAAAACGCCATCACCACGTC-3'

cyclinD1 Forward: 5'-GATGCCAACCTCCTCAACGA-3' Reverse: 5'-ACTTCTGTTCCTCGCAGACC-3'

c-Myc Forward: 5'-CCCTCCACTCGGAAGGACTA-3' Reverse: 5'-GCGGTGCATTTTCGGTTGT-3'

GAPDH Forward: 5'-CATCACCATCTTCCAGGAGCG-3 Reverse: 5'-TGACCTTGCCCACAGCCTTG-3'

TRIM24, tripartite motif-containing 24; GAPDH, glyceraldehyde-3-phosphate dehydrogenase.

of TRIM24 on Wnt/ $\beta$-catenin signaling, the NC-transfected and TRIM24 siRNA-transfected cells were incubated with the Wnt/ $\beta$-catenin signaling activator, lithium chloride $(\mathrm{LiCl}$; $20 \mathrm{mmol} / \mathrm{l})$ for $24 \mathrm{~h}$.

Cell proliferation assay. An MTT assay was used to assess cell proliferation, according to the manufacturer's protocol. Briefly, the NC-transfected and TRIM24 siRNA-transfected cells were seeded into 96 -well plates at a density of $1 \times 10^{3}$ cells/ well. Following various durations (1-5 days), $20 \mu 1$ MTT (Sigma-Aldrich; Merck Millipore) was added to each well and incubated at $37^{\circ} \mathrm{C}$ for $4 \mathrm{~h}$; dimethyl sulfoxide $(150 \mu \mathrm{l}$; Sigma-Aldrich; Merck Millopore) was mixed into each well for $10 \mathrm{~min}$, and the absorbance at $490 \mathrm{~nm}$ was measured using a microplate reader (Bio-Rad Laboratories, Inc., Hercules, CA, USA). Each sample had four replicates.

Colony formation assay. The NC-transfected and TRIM24 siRNA-transfected MGC803 and HGC-27 cells $\left(3 \times 10^{2}\right.$ and $4 \times 10^{2} /$ well, respectively) were seeded in four $6-\mathrm{cm}$ dishes and cultured for 2 weeks. Colonies ( $>50$ cells) were visualized using $5 \%$ crystal violet staining and counted. The results are reported as the average of three independent experiments.

Cell cycle and apoptosis analyses. The cells were collected $48 \mathrm{~h}$ following siRNA transfection, and cell cycle phase was determined using flow cytometry (Beckman-Coulter, Inc., Fullerton, CA, USA). The NC-transfected and TRIM24 siRNA-transfected cells were washed in cold PBS and fixed overnight in $1 \mathrm{ml}$ of $70 \%$ ethanol. The following day, the cells were collected, washed and stained with propidium iodide (PI) for $30 \mathrm{~min}$ at $4^{\circ} \mathrm{C}$. Apoptosis was quantified using an Annexin V-Fluorescein Isothiocyanate Apoptosis Detection kit (Sigma-Aldrich; Merck Millipore) according to the manufacturer's instructions. Briefly, $1 \times 10^{6} \mathrm{NC}$-transfected and TRIM24 siRNA-transfected cells were collected $48 \mathrm{~h}$ following transfection, washed twice in cold PBS, resuspended in $500 \mu 1$ binding buffer, incubated with Annexin V-PI for
15 min at room temperature and analyzed using flow cytometry.

Transwell migration and invasion assays. The cells were seeded into 24-well Transwell plates with a pore size of $8 \mu \mathrm{m}$ (Corning Incorporated, Corning, NY, USA). The upper chamber was either left uncoated for the migration assay or precoated with Matrigel for the invasion assay. For the migration assay, the

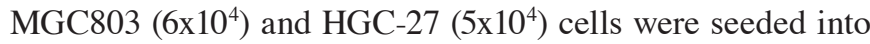
the upper chamber; for the invasion assay, $1 \times 10^{5}$ MGC803 and $1 \times 10^{5}$ HGC-27 cells were seeded into the upper chamber, respectively. The upper chamber was filled with RPMI 1640 medium containing $10 \mathrm{~g} / 1$ BSA (Sigma-Aldrich; Merck Millipore) and the lower chamber was filled with RPMI 1640 containing 25\% FBS. Following incubation for 24 or $36 \mathrm{~h}$ at $37^{\circ} \mathrm{C}$, the cells that had invaded into the lower chamber were fixed with $4 \%$ paraformaldehyde and stained with crystal violet for $1 \mathrm{~h}$ at room temperature, and counted in five randomly-selected microscopic fields. All these experiments were performed in triplicate.

Immunofluorescence. The cells were cultured in confocal dishes and fixed in $4 \%$ paraformaldehyde for $20 \mathrm{~min}$ washed three times with PBS and then permeabilized with $0.2 \%$ Triton $\mathrm{X}-100$ for $10 \mathrm{~min}$, all at room temperature. Following blocking with 5\% BSA at room temperature for $2 \mathrm{~h}$, the cells were incubated with rabbit anti-human monoclonal anti- $\beta$-catenin antibody (1:200 dilution; catalog no. 8480; Cell Signaling Technology, Inc.) overnight at $4^{\circ} \mathrm{C}$. Following washing in PBS three times, the cells were incubated with appropriate Alexa Fluor 488-conjugated secondary antibodies (1:500 dilution; catalog no. ab150077; Abcam) for $1 \mathrm{~h}$ at room temperature, and then counterstained with 4',6-diamidino-2-phenylindole for $10 \mathrm{~min}$. Fluorescence images were captured using laser confocal microscopy.

Statistical analysis. Data were analyzed using paired t-tests to compare quantitative variables. Differences in the expression of TRIM24 between tumor and normal tissues were compared using the Mann-Whitney $\mathrm{U}$ test. $\chi^{2}$ tests were used to assess the correlation between the expression of TRIM24 and clinicopathological characteristics. Survival curves were plotted using the Kaplan-Meier method, and OS rates were compared using the log-rank test. All analyses were performed using SPSS version 18.0 software (SPSS, Inc., Chicago, IL, USA). $\mathrm{P}<0.05$ was considered to indicate a statistically significant difference.

\section{Results}

TRIM24 is overexpressed in GC cell lines and tissues. To investigate the role of TRIM24 in the progression of GC, the present study examined the protein levels of TRIM24 in five human GC cell lines (AGS, BGC823, MGC803, HGC-27 and SGC7901) and one normal gastric cell line (GES-1). TRIM24 was expressed in all cell GC lines, being particularly high in the MGC803 and HGC-27 cells, and low in the AGS cells, compared with the level in the GES-1 cells (Fig. 1A). The expression of TRIM24 was then randomly detected in four pairs of GC tissues and adjacent non-tumor tissues, it was found that the expression of TRIM24 was higher in the 
A

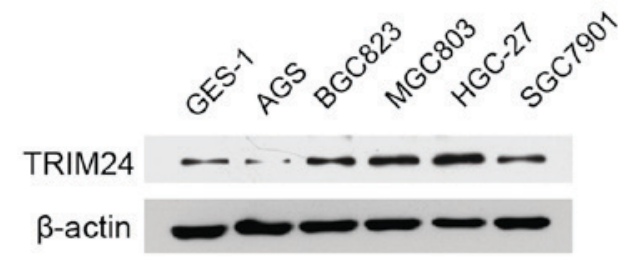

B

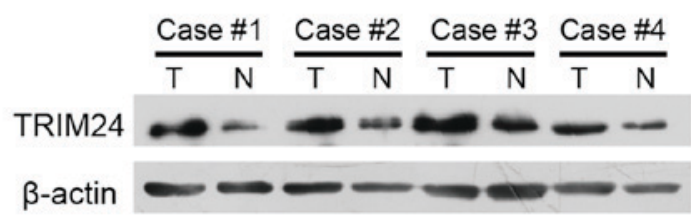

C

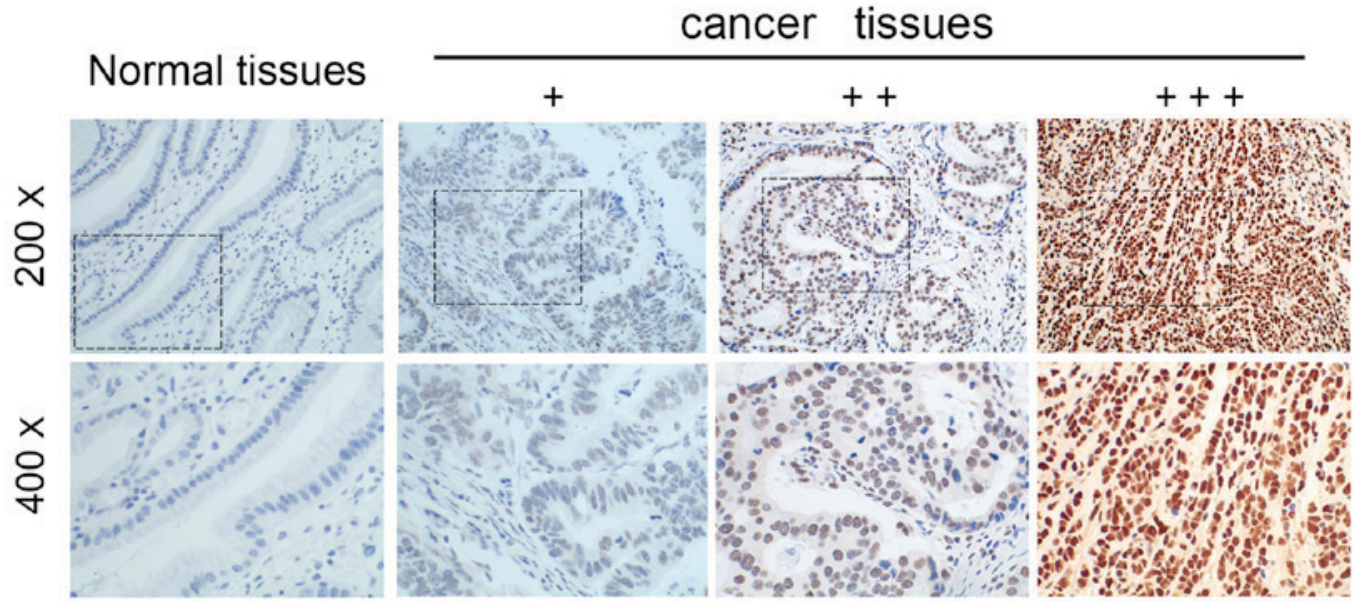

D

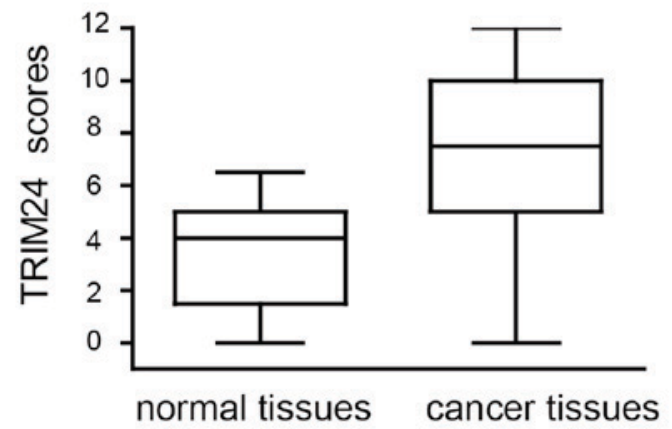

$\mathbf{E}$

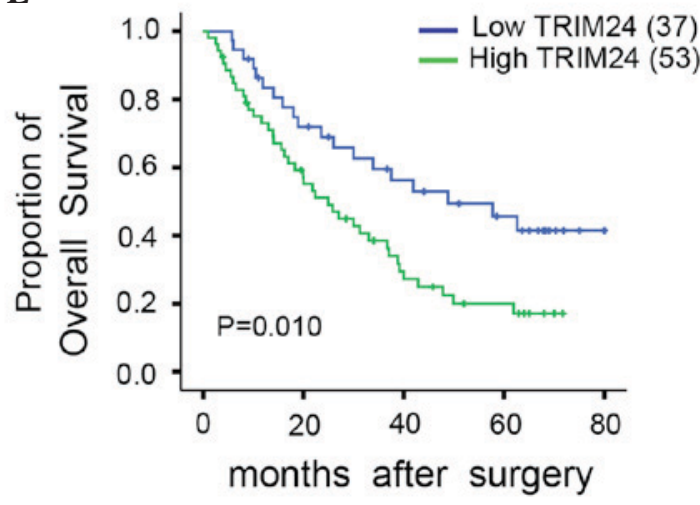

Figure 1. TRIM24 is upregulated in GC cell lines and primary human GC tissues. (A) Western blot analysis of the expression of TRIM24 in five GC cell lines and the GES-1 normal immortal gastric cell line. (B) Western blot analysis of the expression of TRIM24 in four paired primary GC and adjacent non-cancerous tissue samples from the same patient. (C) Expression of TRIM24 in human GC (+, ++ and +++) and normal gastric tissues, visualized using immunohistochemistry (magnification, x100). (D) Expression scores of TRIM24 are shown as box plots, with horizontal lines indicating the median. (E) Kaplan-Meier survival curves of patients with GC stratified by low $(n=37)$ and high $(n=53)$ expression levels of TRIM24 (P=0.01). TRIM24, tripartite motif-containing 24; GC, gastric cancer; $\mathrm{T}$, tumor tissue; $\mathrm{N}$, non-cancerous tissue.

tumor tissues, compared with the adjacent non-tumor tissues (Fig. 1B). The present study subsequently performed immunohistochemistry on 60 normal gastric tissues and 90 primary GC tumor tissues to detect the expression levels of TRIM24. It was found that TRIM24 was expressed in the nuclei of the tumor cells, whereas the normal tissues exhibited negative or weak nuclear staining (Fig. 1C). A significant upregulation in the expression of TRIM24 was observed in 58.9\% (53/90) of the GC tissues, compared with the normal tissues, and this difference was statistically significant ( $\mathrm{P}<0.001$; Fig. 1D).

The present study investigated the association between the expression of TRIM24 and the clinicopathological features of GC (Table I). A high expression level of TRIM24 was correlated with depth of invasion $(\mathrm{P}=0.007)$, tumor-node-metastasis
(TNM) stage $(\mathrm{P}=0.005)$ and lymph node metastasis $(\mathrm{P}=0.027)$. However, no correlations were found between the expression of TRIM24 and other variables, including age $(\mathrm{P}=0.848)$, gender $(\mathrm{P}=0.294)$, tumor size $(\mathrm{P}=0.571)$, location $(\mathrm{P}=0.605)$, tumor differentiation $(\mathrm{P}=0.176)$ or Lauren classification $(\mathrm{P}=0.551)$. Kaplan-Meier analysis revealed that $\mathrm{OS}$ rates were lower in patients with high levels of TRIM24, compared with those with low levels ( $\mathrm{P}=0.010$; Fig. 1E; log-rank test). These data indicated that TRIM24 was overexpressed in GC cell lines and tissues, which was associated with a more malignant phenotype and poorer prognosis in patients with GC.

Knockdown of TRIM24 inhibits proliferation and induces apoptosis of GC cells. To investigate the function of TRIM24 
A

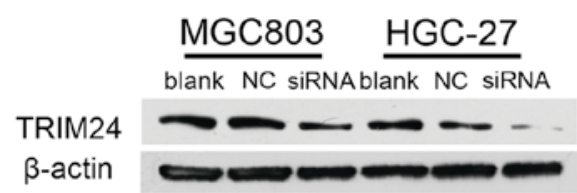

C
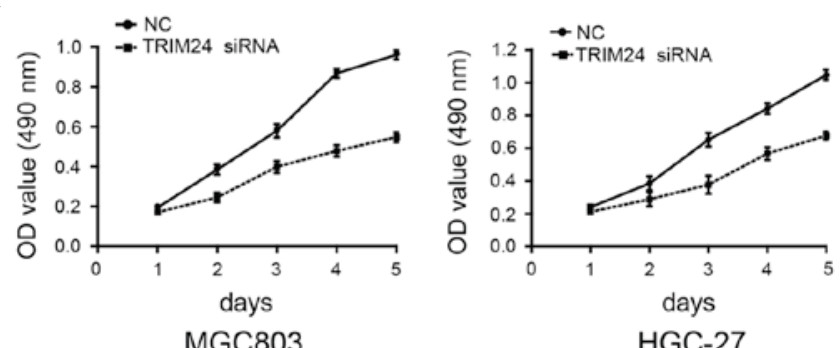

HGC-27

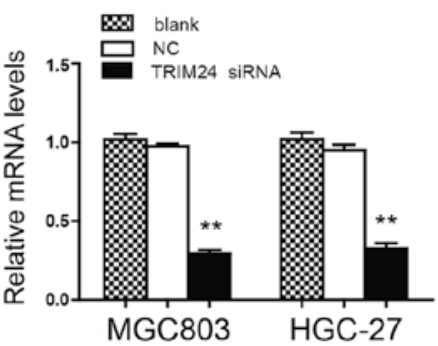

D
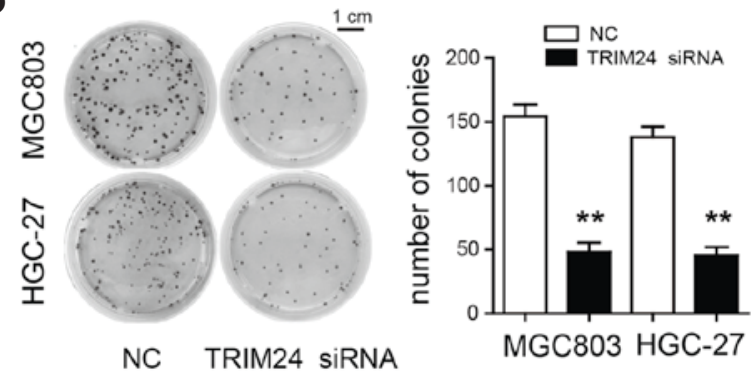

$\mathbf{E}$
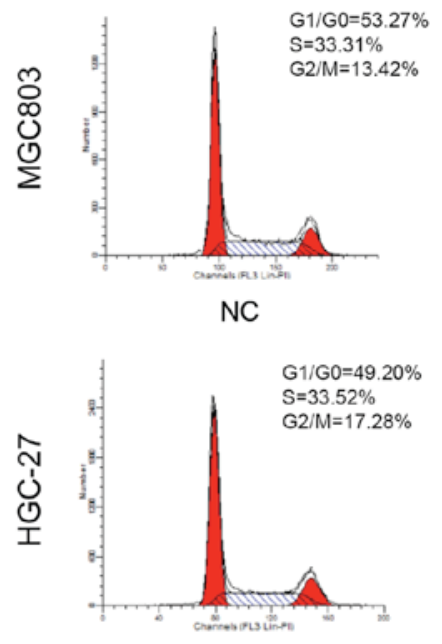

NC

F

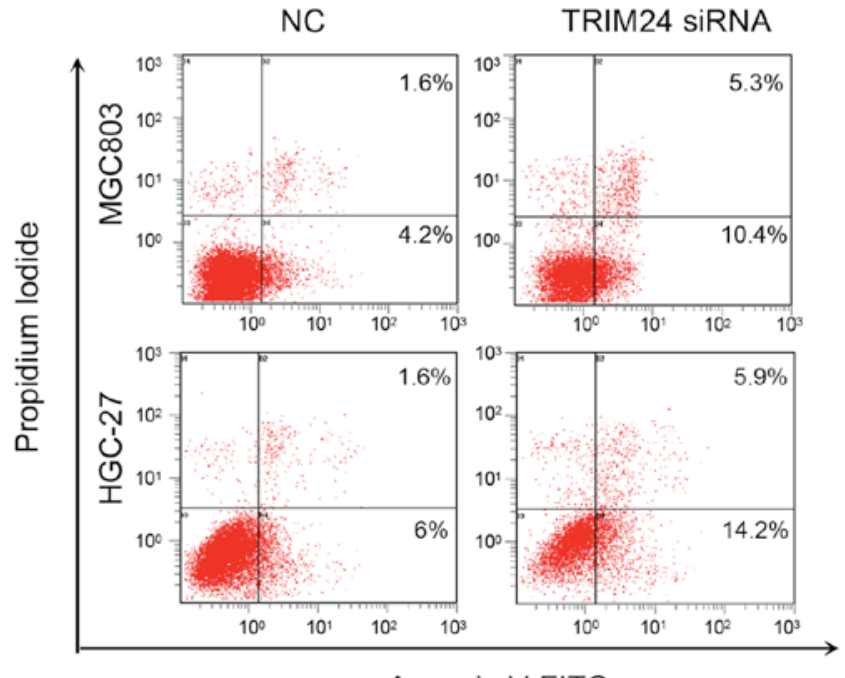

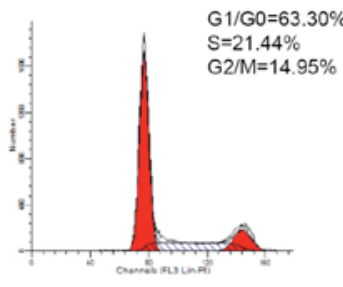

TRIM24 siRNA

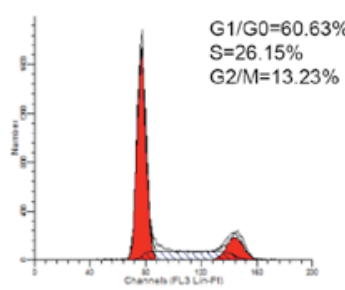

TRIM24 siRNA
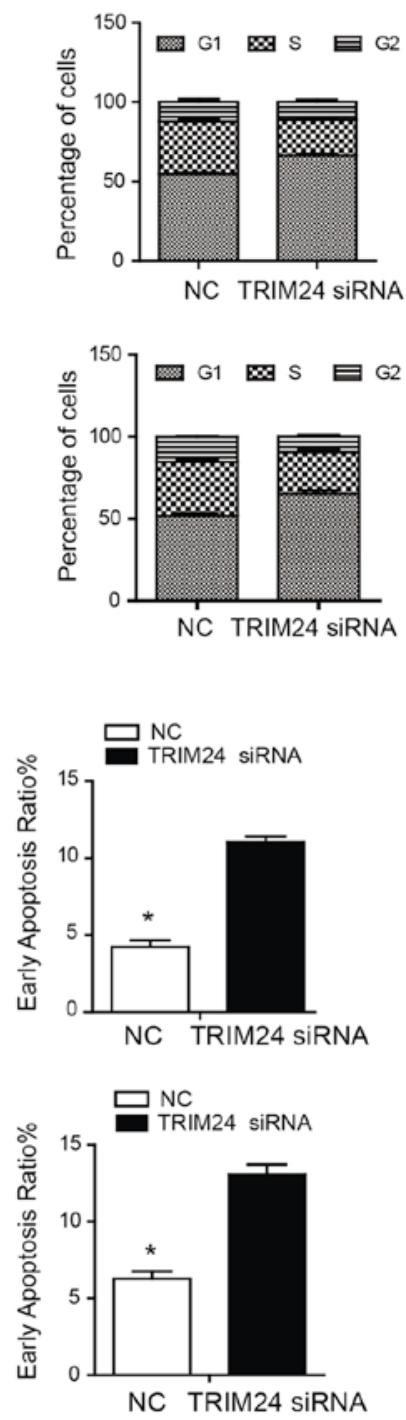

Figure 2. Knockdown of TRIM24 inhibits GC cell proliferation and induces apoptosis. (A) TRIM24 knockdown in MGC803 and HGC-27 cells, evaluated using western blot analysis. $\beta$-actin was a loading control. (B) mRNA expression of TRIM24 transfected with NC or TRIM24 siRNA, normalized against GAPDH. (C) Viability of cells transfected with NC or TRIM24 siRNA, determined using an MTT assay. (D) Growth of cells transfected with NC or TRIM24 siRNA, determined with a colony formation assay. (E) Flow cytometric analysis of cell cycle in GC cells transfected with NC or TRIM24 siRNA. (F) Apoptosis of GC cells, determined using flow cytometry. Data are shown as the mean \pm standard deviation of three independent experiments. "P $<0.05$; ${ }^{* * *} \mathrm{P}<0.01$. TRIM24, tripartite motif-containing 24; GC, gastric cancer; siRNA, small interfering RNA; NC, negative control; OD, optical density; FITC, fluorescein isothiocyanate. 
A
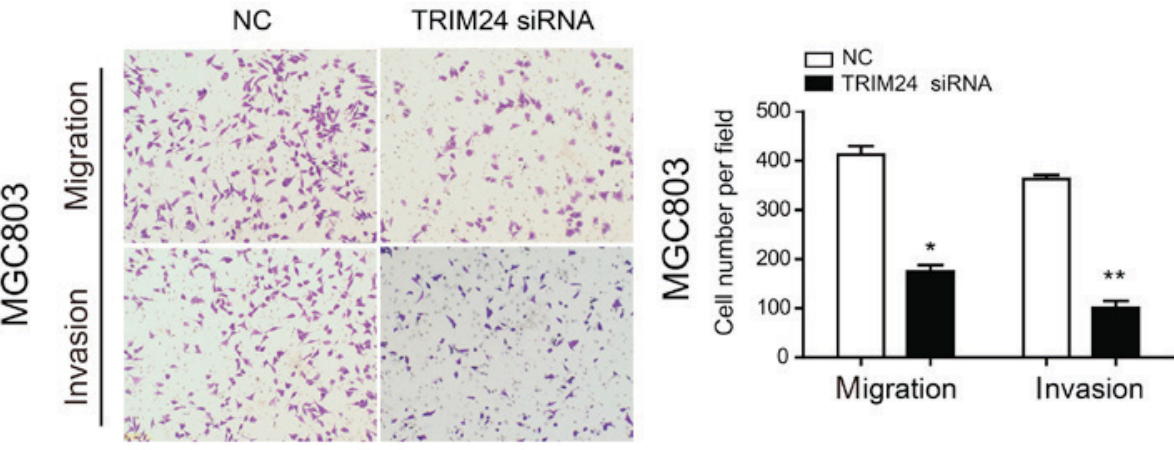

B
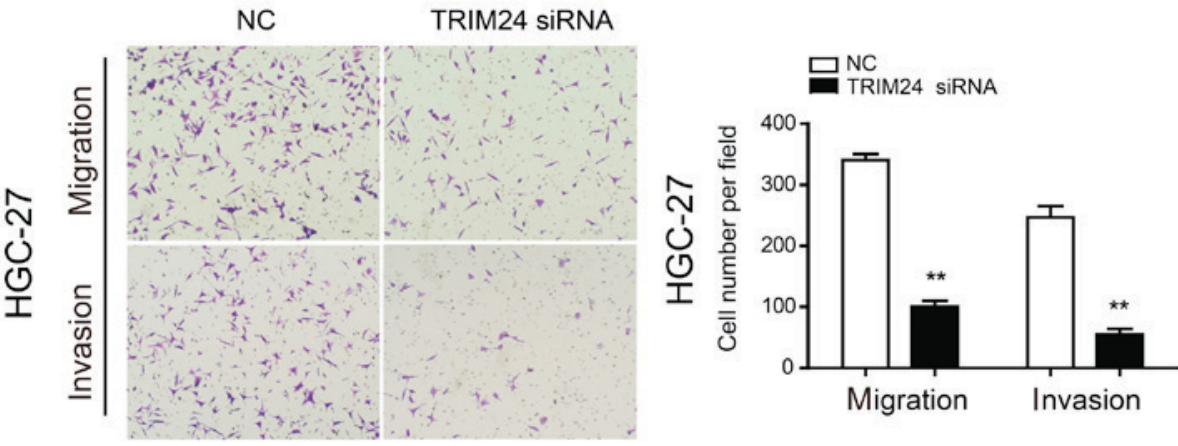

Figure 3. Knockdown of TRIM24 suppresses GC cell migration and invasion. Migration and invasion of (A) MGC803 and (B) HGC-27 cells transfected with NC or TRIM24 siRNA were evaluated using Transwell assays. Data are presented as the mean + standard deviation of three independent experiments. ${ }^{*} \mathrm{P}<0.05$; ${ }^{* *} \mathrm{P}<0.01$. TRIM24, tripartite motif-containing 24; GC, gastric cancer; siRNA, small interfering RNA; NC, negative control.

in GC, the present study evaluated the effects of TRIM24 knockdown on the proliferation and apoptosis of MGC803 and HGC-27 cells. The mRNA and protein levels of TRIM24 were decreased following transfection with TRIM24 siRNA (Fig. 2A and B). TRIM24 knockdown inhibited proliferation, as determined using the MTT and colony formation assays (Fig. 2C and D). Flow cytometric analysis revealed a decrease in the percentage of cells in the $\mathrm{S}$ phase and an increase in the percentages of $\mathrm{G} 1 / \mathrm{G} 0$ phase cells, suggesting that TRIM24 knockdown induced G1/S arrest (Fig. 2E). Moreover, TRIM24 knockdown increased early apoptosis in the MGC803 (10.4\%) and HGC-27 (14.2\%) cells, compared with the control MGC803 (4.2\%) and HGC-27 (6\%) cells (Fig. 2F). These results indicated that TRIM24 had an oncogenic role in the GC cells.

Knockdown of TRIM24 suppresses GC cell migration and invasion. As the expression of TRIM24 was correlated with lymph node metastasis, the present study performed Transwell migration and invasion assays to evaluate the role of TRIM24 in these processes. TRIM24 knockdown inhibited the migration and invasion of MGC803 and HGC-27 cells, compared with the control cells (Fig. 3A and B), demonstrating that TRIM24 promoted GC cell migration and invasion.

TRIM24 regulates the Wnt/ $\beta$-catenin signaling pathway. Aberrant activation of the $\mathrm{Wnt} / \beta$-catenin signaling reportedly contributes to the development and progression of GC $(2,24)$. Therefore, the present study examined the association between the expression of TRIM 24 and $\beta$-catenin in patients with GC. It was found that $\beta$-catenin was accumulated predominantly in the nuclei of the GC cells, and a higher level of TRIM24 was associated with elevated nuclear expression of $\beta$-catenin (Fig. 4A). To further investigate the correlation between TRIM2 4 and $\beta$-catenin, immunohistochemical analysis was used to detect the expression of TRIM24 and $\beta$-catenin in the same sample. Correlation analysis revealed that TRIM24 was positively associated with the nuclear expression of $\beta$-catenin; $\beta$-catenin positive staining was observed in 43 of the high TRIM24-expressing tumors, but only in nine of the low TRIM24-expressing tumors $(\mathrm{P}<0.001 ; \mathrm{r}=0.566$; Fig. 4B).

To clarify the role of $\mathrm{Wnt} / \beta$-catenin signaling in the TRIM24-mediated progression of GC, the present study examined the expression levels of downstream genes in the Wnt/ $\beta$-catenin signaling pathway following TRIM24 knockdown using RT-qPCR and western blot analyses. The knockdown of TRIM24 resulted in the downregulation of $\beta$-catenin, cyclinD1 and c-Myc at the mRNA and protein levels (Fig. 4C and D). Furthermore, TRIM24 knockdown significantly reduced nuclear $\beta$-catenin accumulation in MGC803 and HGC-27 cells, compared with the control groups, as detected using immunofluorescent staining (Fig. 4E), indicating that the knockdown of TRIM24 inhibited the Wnt/ $\beta$-catenin pathway through preventing the nuclear translocation of $\beta$-catenin.

To further confirm the above findings, $\mathrm{LiCl}$, which inhibits GSK-3 $\beta$ activity, was used to activate $\mathrm{Wnt} / \beta$-catenin signaling in GC cells. $\mathrm{LiCl}$ treatment induced the upregulation of $\beta$-catenin, cyclinD1 and c-Myc, and also abrogated the effects of TRIM24 knockdown on the Wnt/ $\beta$-catenin pathway (Fig. 4F). These data suggested that TRIM24 functioned as a positive regulator of $\mathrm{Wnt} / \beta$-catenin signaling, which consequently resulted in an aggressive phenotype in GC. 
A

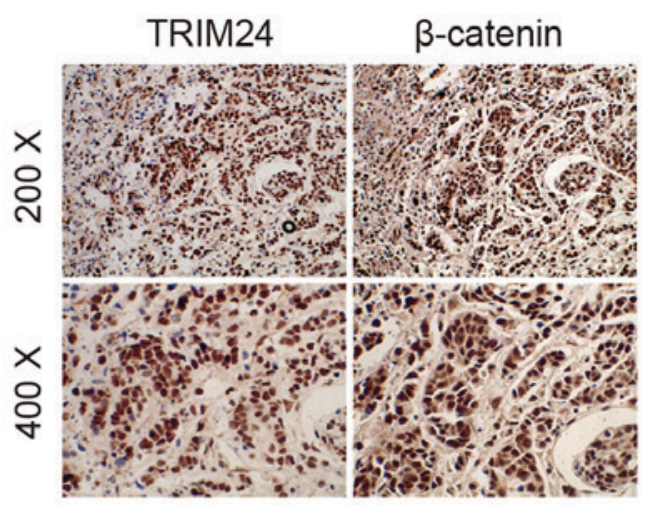

C

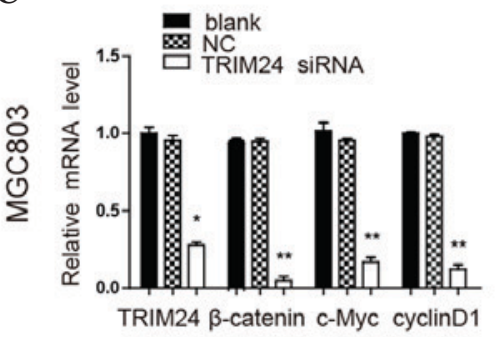

$\mathbf{E}$

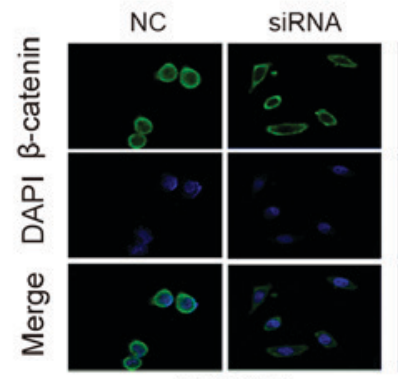

MGC803

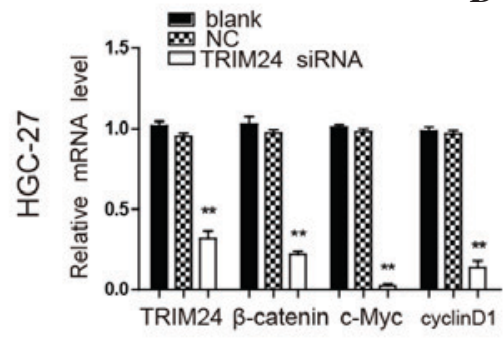

F

Figure 4. TRIM24 regulates the Wnt/ $\beta$-catenin signaling pathway. (A) Representative images of GC tissues showing concordant positive staining of TRIM24 and $\beta$-catenin in the same sample. (B) Quantitative analysis of expression levles of TRIM24 and $\beta$-catenin in 90 GC samples. (C) mRNA expression levels of TRIM24, $\beta$-catenin, c-Myc and cyclinD1 in GC cells transfected with NC or TRIM24 siRNA were analyzed using reverse transcription-quantitative polymerase chain reaction analysis, with GAPDH used as an internal control. (D) Protein expression levels of TRIM24, $\beta$-catenin, c-Myc and cyclinD1 in GC cells transfected with NC or TRIM24 siRNA, as determined using western blot analysis. $\beta$-actin was used as internal loading control. (E) Immunofluorescence staining of subcellular localization of $\beta$-catenin in cells. (F) Protein levels of TRIM24, $\beta$-catenin, c-Myc and cyclinD1 in GC cells transfected with NC or TRIM24 siRNA (+/- $20 \mathrm{mmol} / 1 \mathrm{LiCl}$ for $24 \mathrm{~h})$, using western blot analysis. Data are presented as the mean \pm standard deviation of three independent experiments. ${ }^{*} \mathrm{P}<0.05 ;{ }^{* *} \mathrm{P}<0.01$. TRIM24, tripartite motif-containing 24; GC, gastric cancer; siRNA, small interfering RNA; NC, negative control.

\section{Discussion}

In the present study, it was demonstrated that TRIM24 was overexpressed in GC cells and tissues, and its high expression was correlated with tumor invasion, advanced TNM stage, lymph node metastasis and shorter survival rates. Through functional investigations, it was found that the downregulation of TRIM24 suppressed cell proliferation, migration and invasion, delayed cell cycle progression and induced apoptosis. Furthermore, a positive correlation was found between the expression of TRIM 24 and $\beta$-catenin in GC tissues, and TRIM24 exerted its oncogenic effects by positively regulating the Wnt/ $\beta$-catenin signaling pathway.

Previous studies have shown that TRIM24 is an oncogene, focused on promoting tumor growth, migration and metastasis. The overexpression of TRIM24 has been reported in several types of human tumor, and has been associated with increased malignant behavior and poor prognosis in cancer, including hepatocellular carcinoma (19), breast cancer (25), head and neck squamous cell carcinoma (26), glioma (27) non-small cell lung cancer (28) and bladder cancer (29). In the present study, the immunohistochemical analysis showed that TRIM24 was significantly overexpressed in GC tissues. A high expression level of TRIM24 was closely associated with the depth of invasion, advanced TNM stage and increased lymph node metastasis, indicating that TRIM24 functioned as an oncogene to promote the invasion, metastasis and progression of GC. However, no correlation was detected between TRIM24 and tumor differentiation or Lauren classification, which suggested that TRIM24 may not be involved in GC differentiation. The finding that high expression levels of TRIM24 were associated with poor survival rates suggested that TRIM24 was critical in the pathogenesis and development of GC and may serve as a prognostic biomarker. 
Previous studies investigated the role of TRIM24 in the progression of tumorigenesis and the mechanisms by which TRIM24 exerts its oncogenic functions. It has been reported that the downregulation of TRIM24 results in the inhibition of cell proliferation combined with a significant enhancement of apoptosis through the modulation of $B$ cell lymphoma-2, caspase 3 and poly (ADP-ribose) polymerase in colorectal cancer cells (30). TRIM24 binds to the phosphoinositide 3-kinase (PI3K) promoter to activate PI3K/Akt signaling, leading to the upregulation of downstream targets, including nuclear factor $-\kappa \mathrm{B}$, and the induction of cell proliferation and chemoresistance (27). In addition, TRIM24 has been reported to interact with p53 and control the level of phosphorylated p53 in an autoregulatory feedback loop (31). The previous studies, demonstrated that TRIM24 can modify cell proliferation, migration, invasion and apoptosis. These findings, in addition to the observations of the present study, demonstrated how TRIM24 promotes the malignant behavior of cancer cells.

The aberrant activation of $\mathrm{Wnt} / \beta$-catenin signaling is associated with tumorigenesis in GC. Of note, the present study found that TRIM2 4 and nuclear $\beta$-catenin were often concomitantly overexpressed in GC tissues, suggesting a regulatory role for TRIM24 in $\beta$-catenin signaling. This was also supported by the observation that TRIM24 knockdown decreased the expression levels of the Wnt/ $\beta$-catenin target genes, $\beta$-catenin, cyclinD1 and c-Myc. CyclinD1 is overexpressed in several types of cancer and serves as a regulator of cell cycle progression through the G1/S checkpoint (32-34). c-Myc is a nuclear phosphoprotein, which regulates the cell cycle and apoptosis (35). Thus, the inhibition of cell proliferation and enhancement of apoptosis resulting from TRIM24 knockdown may be partly due to the downregulation of cyclinD1 and c-Myc. The Wnt/ $\beta$-catenin pathway is fundamental in the epithelial-mesenchymal transition (EMT), an important step in oncogenic transformation, which includes cell invasion and metastasis (36-38). The nuclear translocation of $\beta$-catenin leads to the downregulation of E-cadherin and subsequent induction of EMT (39), whereas the upregulation of $\beta$-catenin is associated with the invasion and metastasis of several types of solid tumor (40-42). A previous study found that TRIM24 knockdown decreased the levels of EMT-associated factors in hepatocellular carcinoma. The findings of the present study showed that TRIM24 promoted GC cell migration and invasion, and was associated with translocation of $\beta$-catenin to the nucleus. This functional analysis suggested that TRIM24 was a crucial factor involved in multiple aspects of the progression of GC.

A previous study reported that TRIM24 may serve as a predictor of survival rates in patients with GC, and that TRIM24 enhances cell proliferation and chemoresistance via Akt signaling activation (43). However, the present study confirmed the critical role of TRIM24 in cell cycle progression, apoptosis and invasion. In addition, the present study was the first, to the best of our knowledge to demonstrate that TRIM24 activated Wnt/ $\beta$-catenin signaling, which also provided novel support for activation of the $\mathrm{Wnt} / \beta$-catenin pathway in GC. However, the precise mechanism between TRIM2 4 and $\beta$-catenin remains to be elucidated. Ubiquitination, microRNA dysregulation and DNA methylation all contribute to dysregulation of the $\mathrm{Wnt} / \beta$-catenin pathway in several tumor types (44-46). Therefore, additional studies are required to elucidate the mechanistic basis of how TRIM24 interacts with $\beta$-catenin in GC.

In conclusion, the present study demonstrated that TRIM24 was upregulated in human GC cells and tissues, and its overexpression was closely associated with increased malignancy and poor prognosis. Furthermore, the functional and mechanistic investigations of TRIM24 performed in the present study indicated that TRIM24 may be important in the control of GC aggressiveness, partly by activating the $\mathrm{Wnt} / \beta$-catenin pathway. These findings identified TRIM24 as a potential therapeutic biomarker for patients with GC and demonstrated its importance in activation of the $\mathrm{Wnt} / \beta$-catenin pathway during the progression of GC.

\section{Acknowledgements}

The study was supported by the JiangXi Province Talent 555 Project, the National Natural Science Foundation of China (grant nos. 81160281 and 81441083), the Major Natural Science Foundation of Jiangxi Province (grant no. 20152ACB20024) and the Science and Technology Project of Jiangxi Province (grant no. 20151BBG70228).

\section{References}

1. Siegel R, Desantis C and Jemal A: Colorectal cancer statistics, 2014. CA Cancer J Clin 64: 104-117, 2014.

2. Clevers $\mathrm{H}$ and Nusse R: Wnt/beta-catenin signaling and disease. Cell 149: 1192-1205, 2012.

3. Behrens J, Jerchow BA, Würtele M, Grimm J, Asbrand C, Wirtz R, Kühl M, Wedlich D and Birchmeier W: Functional interaction of an axin homolog, conductin, with beta-catenin, APC, and GSK3beta. Science 280: 596-599, 1998.

4. Ikeda S, Kishida S, Yamamoto H, Murai H, Koyama S and Kikuchi A: Axin, a negative regulator of the Wnt signaling pathway, forms a complex with GSK-3beta and beta-catenin and promotes GSK-3beta-dependent phosphorylation of beta-catenin. EMBO J 17: 1371-1384, 1998.

5. Ogasawara N, Tsukamoto T, Mizoshita T, Inada K, Cao X, Takenaka Y, Joh T and Tatematsu M: Mutations and nuclear accumulation of beta-catenin correlate with intestinal phenotypic expression in human gastric cancer. Histopathology 49: 612-621, 2006.

6. Klaus A and Birchmeier W: Wnt signaling and its impact on development and cancer. Nat Rev Cancer 8:387-398, 2008.

7. Clevers $\mathrm{H}$ : Wnt/ $\beta$-catenin signaling in development and disease. Cell 127: 469-480, 2006.

8. Liu W, Dong X, Mai M, Seelan RS, Taniquchi K, Krishnadath KK, Halling KC, Cunningham JM, Boardman LA, Qian C, Christensen E, Schmidt SS, Roche PC, Smith DI and Thibodeau SN: Mutations in AXIN2 cause colorectal cancer with defective mismatch repair by activating beta-catenin/TCF signaling. Nat Genet 26: 501, 2000 .

9. Hatakeyama S: TRIM proteins and cancer. Nat Rev Cancer 11: 792-804, 2011.

10. Herquel B, Ouararhni K and Davidson I: The TIF1 $\alpha$-related TRIM cofactors couple chromatin modifications to transcriptional regulation, signaling and tumor suppression. Transcription 2: 231-236, 2011.

11. Allton K, Jain AK, Herz HM, Tsai WW, Jung SY, Qin J, Bergmann A, Johnson RL and Barton MC: Trim24 targets endogenous p53 for degradation. Proc Natl Acad Sci USA 106: 11612-11616, 2009.

12. Jain AK and Barton MC: Regulation of p53: TRIM24 enters the RING. Cell Cycle 8: 3668-3674, 2009.

13. Tsai WW, Wang Z, Yiu TT, Tsai WW, Jung SY, Qin J, Bergmann A, Johnson RL, Barton MC, Aronow B, et al: TRIM24 links a non-canonical histone signature to breast cancer. Nature 468: 927-932, 2010. 
14. Pathiraja TN, Thakkar KN, Jiang S, Stratton S, Liu Z, Gagea M, Shi X, Shah PK, Phan L, Lee MH, et al: TRIM24 links glucose metabolism with transformation of human mammary epithelial cells. Oncogene 34: 2836-2845, 2015.

15. Zhong S, Delva L, Rachez C, Cenciarelli C, Gandini D, Zhang H, Kalantry S, Freedman LP, Pandolfi PP, et al: A RA-dependent, tumour-growth suppressive transcription complex is the target of the PML-RARalpha and T18 oncoproteins. Nat Genet 23: 287-295, 1999 .

16. Belloni E, Trubia M, Gasparini P, Micucci C, Tapinassi C, Confalonieri S, Nuciforo P, Martino B, Lo-Coco F, Pelicci PG and Di Fiore PP: 8p11 myeloproliferative syndrome with a novel $t(7 ; 8)$ translocation leading to fusion of the FGFR1 and TIF1 genes. Genes Chromosomes Cancer 42: 320-325, 2005.

17. Klugbauer S and Rabes HM: The transcription coactivator HTIF1 and a related protein are fused to the RET receptor tyrosine kinase in childhood papillary thyroid carcinomas. Oncogene 18 : 4388-4393, 1999.

18. Wang L, Heidt DG, Lee CJ, Yang H, Logsdon CD, Zhang L, Fearon ER, Ljungman $\mathrm{M}$ and Simeone DM: Oncogenic function of ATDC in pancreatic cancer through Wnt pathway activation and beta-catenin stabilization. Cancer Cell 15: 207-219, 2009.

19. Liu X, Huang Y, Yang D, Li X, Liang J, Lin L, Zhang M, Zhong K, Liang B and Li J: Overexpression of TRIM24 is associated with the onset and progress of human hepatocellular carcinoma. PloS One 9: e85462, 2014.

20. Edge SB, Byrd DR, Compton CC, Fritz AG, Greene FL and Trotti A (eds): AJCC Cancer Staging Manual. Springer, New York, NY, 2010.

21. Bosman FT, Carneiro F, Hruban RH and Theise ND: WHO Classification of Tumours of the Digestive System. 4th edition. IARC Press, Lyon, 2010.

22. Pan X, Zhou T, Tai YH, Wang C, Zhao J, Cao Y, Chen $Y$, Zhang PJ, Yu M, Zhen C, et al: Elevated expression of CUEDC2 protein confers endocrine resistance in breast cancer. Nat Med 17: 708-714, 2011.

23. Livak KJ and Schmittgen TD: Analysis of relative gene expression data using real-time quantitative PCR and the 2(-Delta Delta C(T)) Method. Methods 25: 402-408, 2001.

24. Wu WK, Cho CH, Lee CW, Fan D, Wu K, Yu J and Sung JJ: Dysregulation of cellular signaling in gastric cancer. Cancer Lett 295: 144-153, 2010

25. Chambon M, Orsetti B, Berthe ML, Bascoul-Mollevi C, Rodriguez C, Duong V, Gleizes M, Thénot S, Bibeau F, Theillet C and Cavaillès V: Prognostic significance of TRIM24/TIF-1 $\alpha$ gene expression in breast cancer. Am J Pathol 178: 1461-1469, 2011

26. Cui Z, Cao W, Li J, Song X, Mao L and Chen W: TRIM24 overexpression is common in locally advanced head and neck squamous cell carcinoma and correlates with aggressive malignant phenotypes. PloS One 8: e63887, 2013.

27. Zhang LH, Yin AA, Cheng JX, Huang HY, Li XM, Zhang YQ, Han N and Zhang X: TRIM24 promotes glioma progression and enhances chemoresistance through activation of the PI3K/Akt signaling pathway. Oncogene 34: 600-610, 2015.

28. Li H, Sun L, Tang Z, Fu L, Xu Y, Li Z, Luo W, Qiu X and Wang E: Overexpression of TRIM24 correlates with tumor progression in non-small cell lung cancer. PloS One 7: e37657, 2012

29. Xue D, Zhang X, Liu J, Liu J, Li N, Liu C, Liu Y and Wang P. Clinical significance and biological roles of TRIM24 in human bladder carcinoma. Tumour Biol 36: 6849-6855, 2015
30. Wang J, Zhu J, Dong M, Yu H, Dai X and Li K: Knockdown of tripartite motif containing 24 by lentivirus suppresses cell growth and induces apoptosis in human colorectal cancer cells. Oncol Res 22: 39-45, 2014.

31. Jain AK, Allton K, Duncan AD and Barton MC: TRIM24 is a p53-induced E3-ubiquitin ligase that undergoes ATM-mediated phosphorylation and autodegradation during DNA damage. Mol Cell Biol 34: 2695-2709, 2014.

32. Knudsen KE, Diehl JA, Haiman CA and Knudsen ES: Cyclin D1: Polymorphism, aberrant splicing and cancer risk. Oncogene 25: 1620-1628, 2006.

33. Roy PG and Thompson AM: Cyclin D1 and breast cancer. Breast 15: 718-727, 2006.

34. Kishimoto I, Mitomi H, Ohkura Y, Kanazawa H, Fukui N and Watanabe M: Abnormal expression of p16 (INK4a), cyclin D1, cyclin-dependent kinase 4 and retinoblastoma protein in gastric carcinomas. J Surg Oncol 98: 60-66, 2008.

35. Niu Z, Liu H, Zhou M, Wang H, Liu Y, Li X, Xiong W, Ma J, Li X and Li G: Knockdown of c-Myc inhibits cell proliferation by negatively regulating the $\mathrm{Cdk} / \mathrm{Rb} / \mathrm{E} 2 \mathrm{~F}$ pathway in nasopharyngeal carcinoma cells. Acta Biochim Biophys Sin (Shanghai) 47: 183-191, 2015.

36. Kalluri R and Weinberg RA: The basics of epithelial-mesenchymal transition. J Clin Invest 119: 1420-1428, 2009.

37. Howard S, Deroo T, Fujita Y and Itasaki N: A positive role of cadherin in Wnt//3-catenin signalling during epithelial-mesenchymal transition. PloS One 6: e23899, 2011.

38. Huber MA, Kraut N and Beug $\mathrm{H}$ : Molecular requirements for epithelial-mesenchymal transition during tumor progression. Curr Opin Cell Biol 17: 548-558, 2005.

39. Polette M, Mestdagt M, Bindels S, Nawrocki-Raby B, Hunziker W, Foidart JM, Birembaut P and Gilles C: Beta-catenin and ZO-1: Shuttle molecules involved in tumor invasion-associated epithelial-mesenchymal transition processes. Cells Tissues Organs 185: 61-65, 2007.

40. Valenta T, Hausmann G and Basler K: The many faces and functions of $\beta$-catenin. EMBO J 31: 2714-2736, 2012.

41. Miyazawa K, Iwaya K, Kuroda M, Harada M, Serizawa H, Koyanagi Y, Sato Y, Mizokami Y, Matsuoka T and Mukai K: Nuclear accumulation of beta-catenin in intestinal-type gastric carcinoma: Correlation with early tumor invasion. Virchows Arch 437: 508-513, 2000.

42. Chiu CG, Chan SK, Fang ZA, Masoudi H, Wood-Baker R, Jones SJ, Gilks B, Laskin J and Wiseman SM: Beta-catenin expression is prognostic of improved non-small cell lung cancer survival. Am J Surg 203: 654-659, 2012.

43. Miao ZF, Wang ZN, Zhao TT, Xu YY, Wu JH, Liu XY, Xu H, You Y and Xu HM: TRIM24 is upregulated in human gastric cancer and promotes gastric cancer cell growth and chemoresistance. Virchows Arch 466: 525-532, 2015.

44. MacDonald BT, Tamai K and He X: Wnt/beta-catenin signaling: Components, mechanisms and diseases. Dev Cell 17: 9-26, 2009.

45. Ying $\mathrm{Y}$ and Tao Q: Epigenetic disruption of the WNT/ beta-catenin signaling pathway in human cancers. Epigenetics 4: 307-312, 2009

46. Liu Y, Huang T, Zhao X and Cheng L: MicroRNAs modulate the Wnt signaling pathway through targeting its inhibitors. Biochem Biophys Res Commun 408: 259-264, 2011. 